\title{
Blind Adaptive Reduced-Rank Detection for DS-CDMA Signals in Multipath Channels
}

\author{
Yu Song, Member, IEEE, and Sumit Roy, Member, IEEE
}

\begin{abstract}
Blind adaptive multiuser detection for direct sequence code division multiple access (DS-CDMA) signals over static and time-varying intersymbol interference (ISI) limited channels is considered. Blind adaptive detectors must be robustified for ISI channels, when there is significant mismatch between the received signature vector and the transmitted code (assumed known at the receiver). A new low-complexity detector is presented that improves on some recently proposed methods without explicit estimation of the ISI channel. The key innovation is a reduced-rank detector architecture combined with an efficient subspace tracker that yields direct accurate estimation of the desired user's received signature. Several representative simulation examples of detector output signal-to-noise-and-interference ratio (SINR) for fading channels are provided in support of our claims of improved efficacy of the method.
\end{abstract}

Index Terms - Blind adaptive filtering, code division multiple access (CDMA), multipath channels, multiuser detection.

\section{INTRODUCTION}

$\mathbf{L}$ INEAR multiuser detectors have shown considerable potential as pragmatic (lower-complexity) reliable detectors for direct sequence code division multiple access (DS-CDMA) signals in additive white Gaussian noise (AWGN) channels. Currently, considerable current effort is aimed at advancing the design of such multiuser detectors to combat intersymbol interference (ISI) and fading. Receivers for multipath fading channels should be adaptive and preferably blind (i.e., do not require any training sequences in startup) [1]. Of note in this regard is the blind least mean square (LMS) implementation of Honig et al. [2] based on the constrained minimum output energy (CMOE) criterion which yields the minimum mean squared error (MMSE) detector except for a trivial scaling. A recursive least squares (RLS) version of the MMSE detector was presented in [3].

For high-rate CDMA systems, the channel delay spread can be a significant fraction of the symbol period, implying that the resulting ISI is no longer negligible, which is contrary to the assumptions in some recent works [4], [5]. This leads

Manuscript received August 31, 1998. This work was supported by U.S. ARO Grant DAAH04-96-1-0088 and AFOSR Grant F49620-1-0472. This work was presented in part at the Communication Theory Mini-Conference, ICC'99, and SPAWC'99.

Y. Song is with the Division of Engineering, University of Texas at San Antonio, San Antonio, TX 78249 USA.

S. Roy was with the Division of Engineering, University of Texas at San Antonio, San Antonio, TX 78249 USA. He is now with the Department of Electrical Engineering, University of Washington, Seattle, WA 98195 USA.

Publisher Item Identifier S 0733-8716(99)08955-6. to a significant mismatch between the transmitted spreading code (knowledge of which is usually assumed at the receiver) and the actual received signature (which is the convolution of the unknown channel and the spreading code). A natural approach to detector design in such cases is to equip the receiver with a channel estimator as an intermediate step, whose output is used to reconstruct the received signature as was done by a batch processing type estimation scheme in [6]. Expectedly, (statistical) errors in channel estimation degrade the (average) output signal-to-interference-and-noise ratio (SINR) - a common measure of detector performance. Further, the motivation to avoid the additional complexity of channel estimation has led to investigations of adaptive detection by constraining the detector with shifted versions of the transmitted spreading code of the desired user. The constrained minimum variance (CMV) method in [7] entirely bypasses channel estimation and has a low complexity of $2 r^{2}$ flops/update, where $r$ is the length of the observed signal vector. However, the method's poor performance in many circumstances is caused by utilization of only part of the desired signal energy, which is particularly serious in fading channels. Improvements were proposed [8]-[10] at the expense of increased complexity. The methods in [9] and [10] improve the CMV detector by estimating auxiliary vectors other than the channel, while the detector in [8] uses equal gain combination of outputs of $L$ versions of the CMV detector (where $L$ is the desired user's channel length measured in chip durations). For long channels, the increase in complexity in the absence of accurate channel estimation leads to generically suboptimal performance.

Another pertinent structure was presented in [4] and [6] which exploits the subspace structure induced by linear CDMA modulation (and subsequent linear filtering by channel etc. followed by oversampling) which is extracted via a singular value decomposition (SVD), followed by closed-form computation of the detector coefficients. For data-adaptive subspace tracking, [4] employs the projection approximation subspace tracking deflation (PASTd) algorithm [11], which uses iterative deflation to extract an (approximately) orthonormal basis for the signal subspace starting with the largest (signal) eigenvalues/eigenvectors. This leads to accumulation of roundoff estimation errors that result in poor estimates of the small signal eigenvalues and corresponding eigenvectors. Since the closed-form detector of [4] requires inversion of the signal eigenvalues, the effect of estimation errors for small signal eigenvalues is exacerbated, leading to degraded output SINR. 
The batch subspace approach in [12] estimates the detector directly, but is based on minimal eigenvector extraction, which is (especially in an adaptive setting) less desirable than the maximal eigenvector tracking adopted in this paper.

To overcome the difficulties of these existing methods, we [13] propose a blind adaptive detector with direct desired signal estimation (i.e., no intermediate channel estimation) based on the fast subspace tracking (FST) method [14]. Observing that the desired signal lies in the intersection of the received signal subspace and the range of the code matrix of the desired user, we develop an efficient recursive estimator of the desired signal vector using the simple power method [15, Sec. 8.2]. Along with FST-based subspace tracking, we implement a reduced-rank detector that shows significant performance improvement over methods without desired signal (or channel) estimation while reducing complexity as compared to a fullrank detector. Although the detector design is presented for chip-synchronous CDMA for convenience, extensions to the chip-asynchronous case were reported in [16].

Finally, [17] considers a training/decision-directed receiver that iteratively updates a full-order receiver (i.e., does not exploit the subspace structure for dimensionality reduction and hence involves no subspace tracking). Further, [17] primarily emphasizes short channel lengths and fast fading, whereas the natural advantages of our detector are best highlighted for slow fading and long channel as is appropriate for high datarate scenarios. Nevertheless, simulation experience with fast fading with our detector corroborates the general conclusion in [17] and [1] that adaptive detector design for severe (fast) frequency-selective fading channels still remains a major challenge.

The rest of the paper is organized as follows. In Section II, an equivalent synchronous signal model for DS-CDMA systems is outlined. Section III develops a method for identifying the desired signal vector based on batch subspace estimation. The adaptive detector is derived in Section IV, and its performance assessed by several typical simulation examples in Section V. Section VI concludes the paper with final remarks.

\section{Signal AND System Model}

In a $K$-user chip-synchronous DS-CDMA system, the transmitted baseband signal of the $k$ th user is

$$
x_{k}(t)=\sqrt{E_{k}} \sum_{n=-\infty}^{\infty} b_{k}(n) v_{k}\left(t-n T-\tau_{k}\right)
$$

where $T$ is the bit interval, $\tau_{k}$ is the transmission delay (assumed to be integer multiples of chip duration $T_{c}$ ), $E_{k}$ is the transmitted bit energy, and $b_{k}(n) \in\{-1,1\}$ is the $n$th information bit of the $k$ th user. The energy normalized transmitted waveform (i.e., $\int v_{k}^{2}(t) d t=1$ ) is given by

$$
v_{k}(t)=\sum_{i=0}^{N-1} c_{k}(i) \psi\left(t-i T_{c}\right)
$$

where $c_{k}\left(c_{k}(i) \in\{-1,1\}\right)$ is the spreading sequence of the $k$ th user, and $\psi(t)$ is the chip waveform (assumed to be rectangular of duration $T_{c}$, where $N=T / T_{c}$ is the spreading factor).

The signal $x_{k}(t)$ traverses through a channel represented by a linear time-invariant filter, the impulse response of which (plus the transmission delay) is denoted by $h_{k}(t)$. The received signal in the presence of AWGN $w(t)$ is input to a chip matched filter front-end followed by a synchronized chip-rate $\left(1 / T_{c}\right)$ sampler, leading to the discrete time signal

$$
r(n)=\sum_{k=1}^{K} \sqrt{E_{k}} \sum_{i=-\infty}^{\infty} b_{k}(i) s_{k}(n-i N)+w(n)
$$

where $w(n)$ is the sampled AWGN with variance $\sigma_{n}^{2}$. We assume different symbols of the same user, as well as symbols of different users, are uncorrelated. The spreading sequences are repeated periodically in each symbol duration (i.e., length$N$ short codes are used). Since existing CDMA systems based on the IS-95 standard use long spreading codes over many symbols, this model and the subsequent method do not apply to these systems. However, future CDMA systems based on short codes have been proposed for next generation personal communication services (PCS), since they ease implementation of multiuser detection [1], [18].

For user $k$, the received signature waveform $s_{k}$ is the convolution of the spreading sequence $c_{k}$ and the channel $h_{k}$, or

$$
\begin{aligned}
s_{k}(n) & =\sum_{i=0}^{N-1} c_{k}(i) h_{k}(n-i) \\
& =\sum_{i=0}^{L-1} h_{k}(i) c_{k}(n-i)
\end{aligned}
$$

where the channel length $L$ is assumed the same for all users without loss of generality. Then, the channel length in bit duration is $L_{b} \triangleq\lceil L+N-1 / N\rceil$, where $\lceil x\rceil$ is the smallest integer greater than or equal to $x$.

Define the following vectors

$$
\begin{aligned}
\boldsymbol{r}(n) & =[r(n N), r(n N+1), \ldots, r((n+1) N-1)]^{T}, \\
\boldsymbol{h}_{k} & =\left[h_{k}(0), h_{k}(1), \ldots, h_{k}(L-1)\right]^{T}, \\
\boldsymbol{s}_{k} & =[\underbrace{s_{k}(0), s_{k}(1), \ldots, s_{k}(N+L-2), 0, \ldots, 0}_{L_{b} N}]^{T} .
\end{aligned}
$$

Partition $\boldsymbol{s}_{k}$ into $L_{b}$ segments with length $N$

$$
\boldsymbol{s}_{k}^{i}=\left[\begin{array}{c}
s_{k}((i-1) N) \\
s_{k}((i-1) N+1) \\
\vdots \\
s_{k}(i N-1)
\end{array}\right], \quad i=1, \ldots, L_{b} .
$$

The received bit vector is

$$
\begin{aligned}
\boldsymbol{r}(n)= & \sum_{k=1}^{K} \sqrt{E_{k}}\left[\begin{array}{llll}
\boldsymbol{s}_{k}^{L_{b}} & \cdots & \boldsymbol{s}_{k}^{2} & \boldsymbol{s}_{k}^{1}
\end{array}\right]\left[\begin{array}{c}
b_{k}\left(n-L_{b}+1\right) \\
\vdots \\
b_{k}(n-1) \\
b_{k}(n)
\end{array}\right] \\
& +\boldsymbol{w}(n) .
\end{aligned}
$$


Consider an observation interval of $m$ bits. The received signal vector is

$$
\begin{aligned}
& \boldsymbol{r}_{m}(n)=\left[\begin{array}{c}
\boldsymbol{r}(n) \\
\boldsymbol{r}(n+1) \\
\vdots \\
\boldsymbol{r}(n+m-1)
\end{array}\right] \\
& =\underbrace{\left[\begin{array}{lll}
\sqrt{E_{1}} \boldsymbol{S}_{1} & \cdots & \sqrt{E_{K}} \boldsymbol{S}_{K}
\end{array}\right]}_{\boldsymbol{S}} \underbrace{\left[\begin{array}{c}
\boldsymbol{b}_{1}(n) \\
\vdots \\
\boldsymbol{b}_{K}(n)
\end{array}\right]}_{\boldsymbol{b}(n)}+\boldsymbol{w}_{m}(n)
\end{aligned}
$$

where the signature matrix and symbol vector of user $k$ are shown in (9) and (10) at the bottom of the page.

In the above example, $\boldsymbol{w}(n)$ and $\boldsymbol{w}_{m}(n)$ are the corresponding noise vectors, and $m$ is the smoothing factor which is relevant to the channel identifiability issue with the subspace method described later.

Assuming user 1 is the desired user, we have the equivalent synchronous model [1]

$$
\boldsymbol{r}_{m}(n)=\tilde{b}_{0}(n) \boldsymbol{u}_{0}+\sum_{i=1}^{I} \tilde{b}_{i}(n) \boldsymbol{u}_{i}+\boldsymbol{w}_{m}(n) .
$$

The desired symbol is $\tilde{b}_{0}(n)=b_{1}(n)$, while the interfering symbols $\tilde{b}_{i}(n), i=1, \ldots, I$ consist of $\left(L_{b}+m-1\right)(K-$ 1) multiuser interference (MUI) and $L_{b}+m-2$ ISI symbols for a total interfering dimension of $I=\left(L_{b}+m-1\right) K-1$. Letting the transmitted code matrix for the desired user be

$$
\boldsymbol{C}_{1}=\left[\begin{array}{ccc}
c_{1}(0) & & \mathbf{0} \\
\vdots & \ddots & \\
c_{1}(N-1) & & c_{1}(0) \\
& \ddots & \vdots \\
& 0 & c_{1}(N-1)
\end{array}\right]_{m N \times L}
$$

we note that the desired signal vector $\boldsymbol{u}_{0}$ is the $L_{b}$ th column of matrix $\boldsymbol{S}_{1}$, i.e.,

$$
\boldsymbol{u}_{0}=C_{1} \boldsymbol{h}_{1}
$$

and that the remaining columns of $S_{1}, \ldots, S_{K}$ represent the interference vectors.

\section{Subspace-BAsed Desired Signal Estimation}

In this section, we deal with the estimation of the desired signal $\boldsymbol{u}_{0}$. Since $\boldsymbol{u}_{0}$ is the convolution of the spreading code and the channel, it can be reconstructed from the channel estimate [4], [6]. The methods in [4] and [6] both estimate the desired user's channel by seeking the minimal eigenvector of a certain symmetric positive definite matrix and reconstruct the desired signal vector. There are fundamental limitations on the relative accuracy with which the eigenvalues of a matrix can be computed by iterative means, with small eigenvalues being more difficult to compute than larger ones [19]. Our method requires direct recursive estimation of $\boldsymbol{u}_{0}$ as the maximal eigenvector of a certain matrix. It bypasses channel estimation and converges faster than the minimal eigenvector extraction methods.

Construct the data matrix

$$
\begin{aligned}
\boldsymbol{X}(n) & =\left[\begin{array}{llll}
\boldsymbol{r}_{m}(n) & \boldsymbol{r}_{m}(n-1) & \cdots & \boldsymbol{r}_{m}\left(n-N_{c}\right)
\end{array}\right] \\
& =\boldsymbol{S} \boldsymbol{B}(n)+\boldsymbol{W}(n)
\end{aligned}
$$

where

$$
\begin{aligned}
\boldsymbol{B}(n) & =\left[\begin{array}{llll}
\boldsymbol{b}(n) & \boldsymbol{b}(n-1) & \cdots & \boldsymbol{b}\left(n-N_{c}\right)
\end{array}\right] \\
\boldsymbol{W}(n) & =\left[\begin{array}{llll}
\boldsymbol{w}_{m}(n) & \boldsymbol{w}_{m}(n-1) & \cdots & \boldsymbol{w}_{m}\left(n-N_{c}\right)
\end{array}\right] .
\end{aligned}
$$

Taking the SVD of $\boldsymbol{X}^{H}$ (time index $n$ omitted)

$$
\boldsymbol{X}^{H}=\left[\begin{array}{ll}
\boldsymbol{U}_{s} & \boldsymbol{U}_{n}
\end{array}\right]\left[\begin{array}{ll}
\boldsymbol{\Sigma}_{s} & \\
& \boldsymbol{\Sigma}_{n}
\end{array}\right] \underbrace{\left[\begin{array}{l}
V_{s}^{H} \\
\boldsymbol{V}_{n}^{H}
\end{array}\right]}_{\boldsymbol{V}^{H}} .
$$

We assume that $B$ is of full row rank

$$
r_{s}=\left(L_{b}+m-1\right) K
$$

which can be achieved with sufficiently large $N_{c}$. The signal and noise subspace are $\operatorname{ran}\left(\boldsymbol{V}_{s}\right)$ and $\operatorname{ran}\left(\boldsymbol{V}_{n}\right)$, respectively.

Remarks: It is common in subspace CDMA receiver literature (e.g., [4]-[6]) to assume that the signature matrix $S$ is of full column rank $r_{s}$. However, this is unnecessary. In practice, $\boldsymbol{S}$ is usually nearly rank deficient due to the likely fact that some channel coefficients are nearly zero and that channel lengths may vary significantly depending on the multipath conditions. For a rank deficient $\boldsymbol{S}$, we still have $\operatorname{ran}\left(\boldsymbol{V}_{s}\right)=$ $\operatorname{ran}(S)$; the only difference is that the number of columns in $\boldsymbol{V}_{s}$ [i.e., $\operatorname{rank}(\boldsymbol{S})$ ] is smaller than $r_{s}$. The following subspace method can still be applied without exact knowledge of the true signal subspace dimension, since overestimating the signal subspace dimension still allows (generically) the extraction $\boldsymbol{u}_{0}$ with partial knowledge of the noise subspace (due to inflation of the signal subspace). A similar case has been addressed in the original paper on subspace based blind identification [20]. This capability alleviates the need for exact knowledge of the rank of the signal subspace. For simplicity of presentation, we will proceed with full rank assumption for $S$ in the remainder of the paper.

$$
\begin{aligned}
\boldsymbol{S}_{k} & \left.=\left[\begin{array}{ccccccc}
\boldsymbol{s}_{k}^{L_{b}} & \ldots & \boldsymbol{s}_{k}^{2} & \boldsymbol{s}_{k}^{1} & & \\
& \boldsymbol{s}_{k}^{L_{b}} & \cdots & \boldsymbol{s}_{k}^{2} & \boldsymbol{s}_{k}^{1} & & \mathbf{0} \\
\mathbf{0} & & \ddots & \ddots & & \ddots & \\
& & & \boldsymbol{s}_{k}^{L_{b}} & \ldots & \boldsymbol{s}_{k}^{2} & \boldsymbol{s}_{k}^{1}
\end{array}\right]\right\} m \text { block rows } \\
\boldsymbol{b}_{k}(n) & =\left[b_{k}\left(n-L_{b}+1\right), \ldots, b_{k}(n), \ldots, b_{k}(n+m-1)\right]^{T} .
\end{aligned}
$$




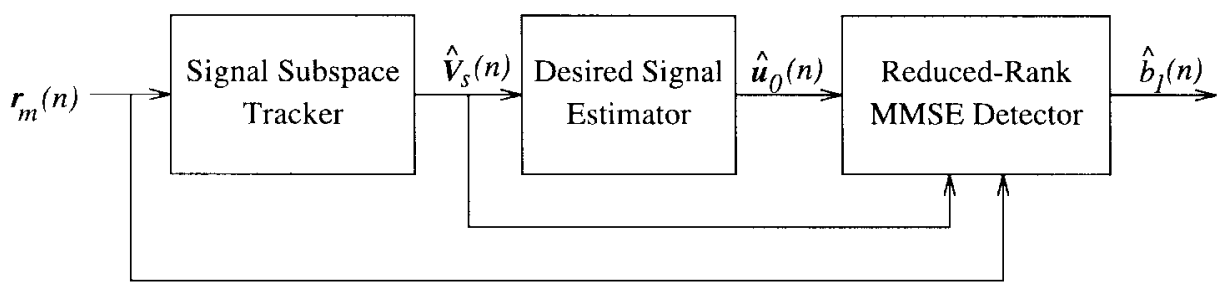

Fig. 1. Blind adaptive detector schematic.

The noise subspace dimension is $r_{n}=r-r_{s}$ and the total dimension is $r=m N$. Due to orthogonality of the signal and noise subspace, and $\operatorname{ran}\left(V_{s}\right)=\operatorname{ran}(S)$, we have

$$
V_{n}^{H} u_{0}=\mathbf{0} .
$$

By defining $\tilde{\boldsymbol{C}}_{1}$ as the orthonormalized version of $C_{1}$ (i.e., as obtained from a Gram-Schmidt procedure)

$$
\begin{aligned}
\boldsymbol{P}_{1} & =\tilde{\boldsymbol{C}}_{1} \tilde{\boldsymbol{C}}_{1}^{H} \\
\boldsymbol{P}_{1}^{\perp} & =\boldsymbol{I}-\boldsymbol{P}_{1}
\end{aligned}
$$

are the orthogonal projection matrices onto $\operatorname{ran}\left(\boldsymbol{C}_{1}\right)$ and $\operatorname{null}\left(\boldsymbol{C}_{1}^{H}\right)$, respectively. From (13), we have

$$
P_{1}^{\perp} u_{0}=\mathbf{0} .
$$

There are $r_{n}=m N-\left(L_{b}+m-1\right) K$ equations in (18) and $m N-L$ equations in (21), while the number of unknowns in $\boldsymbol{u}_{0}$ is $m N$. Imposing constraints (18) and (21) simultaneously, a unique solution for $\boldsymbol{u}_{0}$ exists if

$$
\operatorname{dim}\left[\operatorname{ran}\left(\boldsymbol{V}_{s}\right) \cap \operatorname{ran}\left(\boldsymbol{C}_{1}\right)\right]=1 .
$$

A necessary condition for (22) to hold is that the number of linear equations equals or exceeds the number of unknowns less one, i.e.,

$$
m N \geq L+\left(L_{b}+m-1\right) K-1 .
$$

For $K<N$, condition (23) can always be satisfied by increasing the smoothing factor $m$. Extensive simulations show that (22) is generally satisfied when (23) is used with an overestimate of the dimension of $\boldsymbol{V}_{s}$.

Since in practice we only have estimates of the subspace, (18) and (21) can be solved in the least-squares sense

$$
\min _{\hat{\boldsymbol{u}}_{0}} \hat{\boldsymbol{u}}_{0}^{H}\left(\hat{V}_{n} \hat{\boldsymbol{V}}_{n}^{H}+\boldsymbol{P}_{1}^{\perp}\right) \hat{\boldsymbol{u}}_{0} \text { s.t. }\left\|\hat{\boldsymbol{u}}_{0}\right\|_{2}=1 .
$$

The solution is the minimal eigenvector of the matrix $\hat{\boldsymbol{V}}_{n} \hat{\boldsymbol{V}}_{n}^{H}+$ $\boldsymbol{P}_{1}^{\perp}$. By considering (20) and recalling

$$
\boldsymbol{V}_{s} \boldsymbol{V}_{s}^{H}+\boldsymbol{V}_{n} \boldsymbol{V}_{n}^{H}=\boldsymbol{I}
$$

(24) can be easily transformed into a maximization problem

$$
\max _{\hat{\boldsymbol{u}}_{0}} \hat{\boldsymbol{u}}_{0}^{H} \boldsymbol{Q} \hat{\boldsymbol{u}}_{0} \text { s.t. }\left\|\hat{\boldsymbol{u}}_{0}\right\|_{2}=1
$$

whose solution is the maximal eigenvector of matrix

$$
\boldsymbol{Q} \triangleq \hat{\boldsymbol{V}}_{s} \hat{\boldsymbol{V}}_{s}^{H}+\tilde{\boldsymbol{C}}_{1} \tilde{\boldsymbol{C}}_{1}^{H} .
$$

\section{Subspace Tracking And Reduced Rank Detector}

The adaptive detector schematic is shown in Fig. 1, which highlights the (feedforward) iterative computations involved. In time-varying scenarios, the full SVD in (16) is computationally too expensive $\left[O\left(r^{3}\right)\right]$ for continuous update and is also unnecessary, since only an orthonormal basis of the signal subspace (and not the entire subspace structure) is required for the desired signal estimation. Thus, effective subspace tracking methods with lower complexity can be adopted-while there exist many methods with different complexity/performance tradeoffs, we concentrate on those with minimal complexity $O\left(r r_{s}\right)$. Within this category, we find that the FST method proposed by Rabideau [14] is particularly suited for our detector architecture. It is based on spherically averaged URV decomposition of the exponentially weighted data matrix ( $\boldsymbol{R}$ in [14] corresponds to $\Sigma$ in our notation)

$$
\boldsymbol{\Gamma} \boldsymbol{X}^{H}=\boldsymbol{U} \boldsymbol{\Sigma} \boldsymbol{V}^{H}
$$

where $\boldsymbol{\Gamma}=\operatorname{diag}\left(\beta^{N_{c}-1}, \ldots, \beta, 1\right), \boldsymbol{V}=\left[\boldsymbol{V}_{s} \boldsymbol{V}_{n}\right]$, and the $r \times r_{s}$ matrix $V_{s}$ is an orthonormal basis of the signal subspace. In addition

$$
\Sigma=\left[\begin{array}{cc}
\Sigma_{s} & 0 \\
0 & \bar{\sigma}_{n} \boldsymbol{I}
\end{array}\right]
$$

where $\Sigma_{s}$ is an $r_{s} \times r_{s}$ upper triangular matrix that contains the signal eigenstructure information and $\bar{\sigma}_{n}$ is the averaged noise power. The simplest one in the FST class is the refinement only FST (RO-FST), which consists of the following three steps and is summarized in Table I:

1) noise averaging;

2) QR decomposition updating (where $\Sigma_{a}$ is obtained by reducing the noise subspace to a single column)

$$
\hat{\boldsymbol{\Sigma}}_{a}=\left[\begin{array}{cc}
\boldsymbol{\Sigma}_{s} & \mathbf{0} \\
\mathbf{0} & \bar{\sigma}_{n}
\end{array}\right]
$$

3) refinement (signal and noise subspace separation).

Extensive simulations in [14] show that RO-FST achieves faster tracking than many other subspace tracking methods by concentrating on separation of the signal and noise subspace, instead of obtaining accurate estimates of the singular values by diagonalizing $\boldsymbol{\Sigma}$. Orthonormality of $\hat{V}_{s}$ is preserved by the Givens rotations used in the update, while noise averaging allows low complexity of $10 r r_{s}+9.5 r_{s}^{2}$ flops/update.

While the signal subspace is recursively updated via ROFST, a simple power iteration method [15, Section 8.2] can be used to adaptively solve (26)

$$
\begin{aligned}
\boldsymbol{a}(n) & =\boldsymbol{Q}(n) \hat{\boldsymbol{u}}_{0}(\boldsymbol{n}-\mathbf{1}) \\
\hat{u}_{0}(n) & =\boldsymbol{a}(n) /\|\boldsymbol{a}(n)\|_{2} .
\end{aligned}
$$


TABLE I

RO-FST

$$
\begin{aligned}
& \bar{\sigma}_{n}=\hat{\mathbf{\Sigma}}_{a}\left(r_{s}+1, r_{s}+1\right) \\
& \mathbf{r}_{s}=\hat{\mathbf{V}}_{s}^{H} \mathbf{r}_{m} \\
& \alpha=\left\|\mathbf{r}_{m}-\hat{\mathbf{V}}_{s} \mathbf{r}_{s}\right\|_{2} \\
& \mathbf{v}_{n}=\frac{\mathbf{r}_{m}-\hat{\mathbf{v}}_{s} \mathbf{r}_{s}}{\alpha} \\
& \boldsymbol{\Sigma}_{b}=\left[\begin{array}{c}
\beta \hat{\mathbf{\Sigma}}_{a} \\
\mathbf{r}_{s}^{H}
\end{array}\right]
\end{aligned}
$$

Apply a sequence of Givens rotation to restore $\Sigma_{b}$ to upper triangular, then drop the last row:

$\boldsymbol{\Sigma}_{c}=\mathbf{G}_{1} \boldsymbol{\Sigma}_{b}$

Refinement: Apply 2 sequences of Givens rotation

to zero the last column and last row, respectively:

$$
\begin{aligned}
& \hat{\mathbf{\Sigma}}_{a}=\mathbf{G}_{2} \boldsymbol{\Sigma}_{c} \mathbf{G}_{3} \\
& {\left[\hat{\mathbf{V}}_{s} \mathbf{v}_{n}\right]=\left[\hat{\mathbf{V}}_{s} \mathbf{v}_{n}\right] \mathbf{G}_{3}} \\
& \hat{\mathbf{\Sigma}}_{a}\left(r_{s}+1, r_{s}+1\right)=\left[\frac{\left(N-r_{s}-1\right)\left(\beta \bar{\sigma}_{n}\right)^{2}+\left|\hat{\sigma}^{2}\right|}{N-r_{s}}\right]^{\frac{1}{2}}
\end{aligned}
$$

The computational complexity of (27), (31), and (32) is $4 r\left(r_{s}+L\right)$. Note that $\tilde{C}_{1}$ is fixed, and only the signal subspace matrix $\hat{V}_{s}(n)$ needs to be updated for updating $\boldsymbol{Q}(\boldsymbol{n})$. The convergence rate of the power method is determined by the ratio of the two largest eigenvalues of $\boldsymbol{Q}(\boldsymbol{n})$. This method requires no user-defined parameter (such as step size), and converges faster than methods seeking minimal eigenvectors. The gradient-based minimal eigenvector tracking method of [21] employed in [4] is known to suffer from slow convergence and difficulty in choosing the appropriate step size. Note that the Newton-based method in [21] is equivalent to updating $\boldsymbol{Q}^{-1}(\boldsymbol{n})$ and then estimating the maximal eigenvector by applying the power method to $\boldsymbol{Q}^{-1}(\boldsymbol{n})$.

Since the optimal linear MMSE detector coefficient vector is known to lie in the signal subspace [6], a reduced-rankadaptive detector constrained to the signal subspace can benefit from faster convergence in situations where the smallest signal eigenvalue is significantly greater than the noise eigenvalues, as shown in [22]. Rank reduction also means reduced detector complexity and is natural in this context since the signal subspace is already extracted. The exponentially windowed sample estimate of the autocorrelation matrix of the observation vector is obtained as

$$
\hat{\boldsymbol{R}}=\frac{1}{L_{w}}(\boldsymbol{X} \boldsymbol{\Gamma})(\boldsymbol{X} \boldsymbol{\Gamma})^{H}
$$

where $\left.L_{w}=1-\beta^{2 N_{c}}\right) /\left(1-\beta^{2}\right)$ is the effective window length. Considering (28) and that the linear MMSE detector satisfies [1]

$$
\boldsymbol{R d}=u_{0}
$$

the reduced-rank detector coefficient $d(n)$ is obtained by solving

$$
\hat{\boldsymbol{V}}_{s}(n) \hat{\boldsymbol{\Sigma}}_{s}^{H}(n) \hat{\boldsymbol{\Sigma}}_{s}(n) \hat{\boldsymbol{V}}_{s}^{H}(n) d(n)=\hat{\boldsymbol{u}}_{0}(n)
$$

The complexity of solving the previous equation is $4 r r_{s}+2 r_{s}^{2}$, since $\hat{V}_{s}(n)$ is orthogonal and $\hat{\boldsymbol{\Sigma}}_{s}(n)$ is triangular [15]. Thus the overall complexity of the detector in Fig. 1 is $18 r r_{s}+$ $11.5 r_{s}^{2}+4 r L$.

\section{Simulation AND Discussion}

We present simulation results for near-far limited scenarios in different channel settings. There are four interfering users, each with MUI $=20 \mathrm{~dB}$ above the desired user $(0 \mathrm{~dB})$. Random binary $( \pm 1)$ spreading codes are generated with spreading factor $N=16$ and kept fixed over all simulation runs. AWGN is added to the received signal so that the average bit signal-tonoise ratio (SNR) for the desired user is $22 \mathrm{~dB}$ (corresponding to chip SNR of $10 \mathrm{~dB}$ ). The frequency-selective Rayleigh fading channels are simulated with Hoeher's method [23], where the discrete channel is modeled as a tapped delay line with time-varying coefficients. Omitting the user index, the channel coefficients at time $k$ are given by

$$
\bar{h}(k, n)=\sum_{i=1}^{P} e^{j\left(\theta_{i}+2 \pi f_{d, i} n T\right)} g\left(k T-\tau_{i}\right)
$$

whose time-averaged norm is

$$
n_{h}=\frac{1}{N_{s}} \sum_{n=1}^{N_{s}}\|\bar{h}(k, n)\|
$$

where $N_{s}$ is the number of transmitted symbols, $P$ is the total number of paths, $T$ is the symbol period, and the initial phase of the $i$ th path $\theta_{i}$ is assumed independent between paths and uniformly distributed in $[0,2 \pi]$. The $k$ th tap coefficient at time $n$ is taken as

$$
h(k, n)=\frac{\bar{h}(k, n)}{n_{h}}
$$

so that the fading channel $h$ has average norm of unity.

The Doppler frequency is generated according to

$$
f_{d, i}=f_{d, \max } \cos u_{i}
$$

where $u_{i}$ is a random number uniformly distributed in $[0,2 \pi]$. In the simulations, $P=50$ is used, and the combined impulse response of the transmit and receiver filters $g$ is taken to be a raised cosine pulse with rolloff factor 0.5 . The power delay profile was assumed to be negative exponential

$$
p\left(\tau_{i}\right)=e^{-\tau_{i}}
$$

where $\tau_{i}$ is in microseconds.

Since PASTd performs correlation domain and RO-FST does data domain processing, for equivalent processing window length we set the forgetting factors $\beta_{2}$ (PASTd) $=\beta_{1}^{2}$ (RO-FST). We take the averaged output SINR at the $n$th iteration as the performance measure

$$
\operatorname{SINR}(n)=\frac{\sum_{i=1}^{M_{c}}\left|\boldsymbol{d}_{i}^{H}(n) \boldsymbol{u}_{0}\right|^{2}}{\sum_{i=1}^{M_{c}}\left|\boldsymbol{d}_{i}^{H}(n)\left[\boldsymbol{r}_{m, i}(n)-b_{1}(n) \boldsymbol{u}_{0}\right]\right|^{2}} .
$$




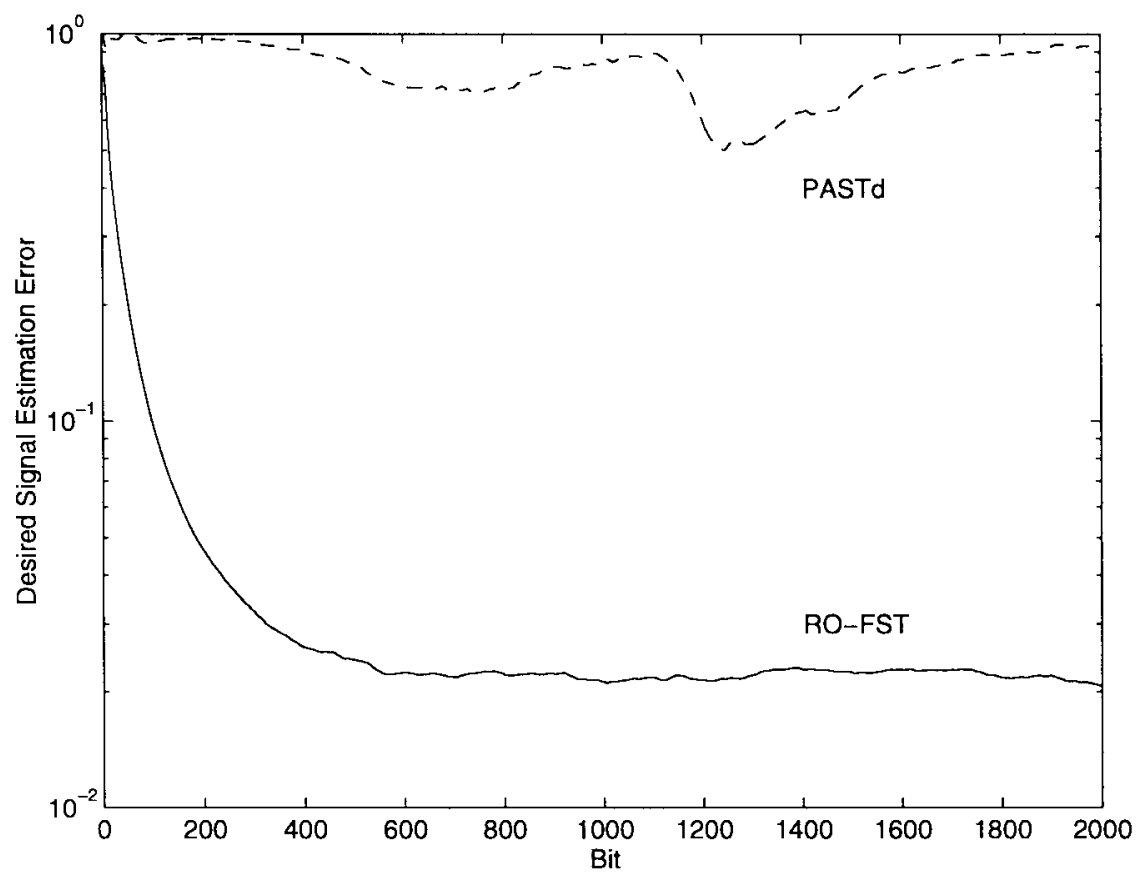

Fig. 2. Performance of desired signal estimators by RO-FST and PASTd, with power-method time-invariant ISI channels, synchronous users; $N=16$, $L=6, m=1$, SNR $=22 \mathrm{~dB}, \beta_{1}=0.997$, and $\beta_{2}=0.994$.

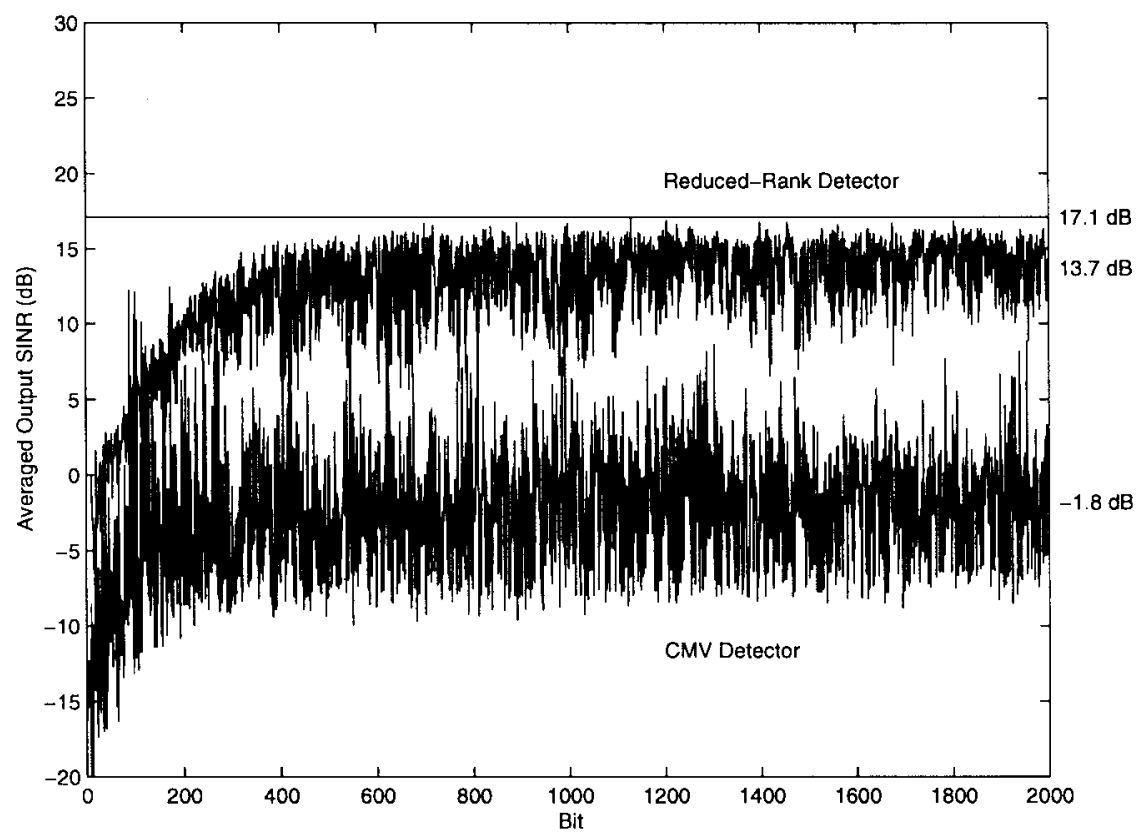

Fig. 3. Performance of the proposed reduced-rank detector and the CMV detector in time-invariant ISI channels, synchronous users; $N=16, L=6$, $m=1, \mathrm{SNR}=22 \mathrm{~dB}, \beta_{1}=0.997$, and $\mu=2 \times 10^{-4}$.

All results shown are based on averages over $M_{c}=100$ Monte Carlo runs. For time-invariant channels, the maximal achievable output SINR of the MMSE detector is also shown, which is calculated from [24]

$$
\begin{aligned}
\operatorname{SINR}_{*} & \triangleq \frac{\mathrm{E}^{2}\left\{\left|\boldsymbol{d}_{\mathrm{MMSE}}^{H} \boldsymbol{r}_{m}(n)\right|\right\}}{\operatorname{var}\left\{\left|\boldsymbol{d}_{\mathrm{MMSE}}^{H} \boldsymbol{r}_{m}(n)\right|\right\}} \\
& =\left|\boldsymbol{u}_{0}^{H}\left(\boldsymbol{R}-\boldsymbol{u}_{0} \boldsymbol{u}_{0}^{H}\right)^{-1} \boldsymbol{u}_{0}\right| .
\end{aligned}
$$

In time-varying examples, we keep the multipath fading channel fixed for each user and perform 100 Monte Carlo runs with different signals and noise. The time-varying SNR (due to fading channel) is plotted for comparison.

Customary initialization is used for all adaptive algorithms. For PASTd, the initial eigenvalues are set to one, and for ROFST, $\hat{\Sigma}_{a}(0)=0$. The initial eigenvectors are chosen to be vectors $\left\{\boldsymbol{e}_{i}\right\}$ (the $i$ th element is one and all others are zero) for both RO-FST and PASTd. The initial desired signal estimate is $\hat{\boldsymbol{u}}_{0}(0)=\boldsymbol{e}_{1}$.

Example 1 (Time-Invariant ISI Channels, Synchronous Users): The data rate is set to $1 \mathrm{Mbit} / \mathrm{s}$, and the channels are time invariant (maximum Doppler frequency $f_{d, \max }=0$ ) 


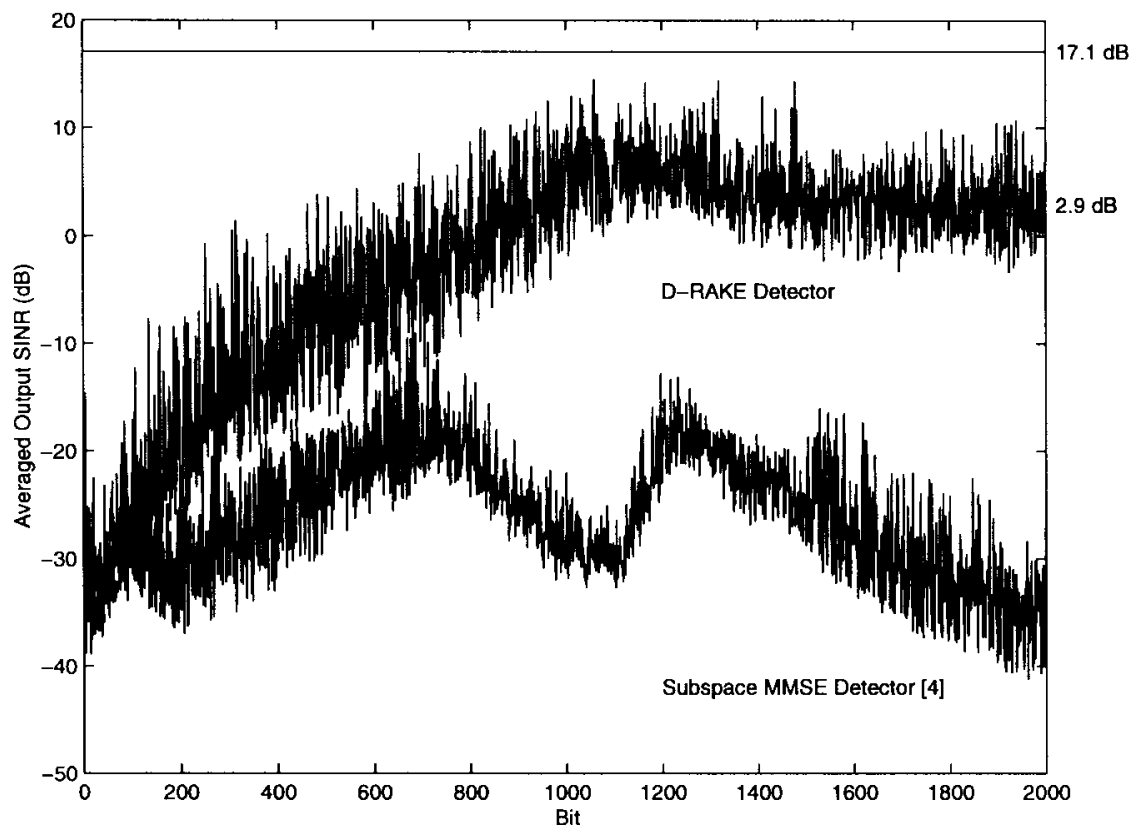

Fig. 4. Performance of the D-RAKE detector and the subspace MMSE detector [4] in time-invariant ISI channels, synchronous users; $N=16, L=6$, $m=1, \mathrm{SNR}=22 \mathrm{~dB}, \beta_{2}=0.994$, and $\mu=2 \times 10^{-4}$.

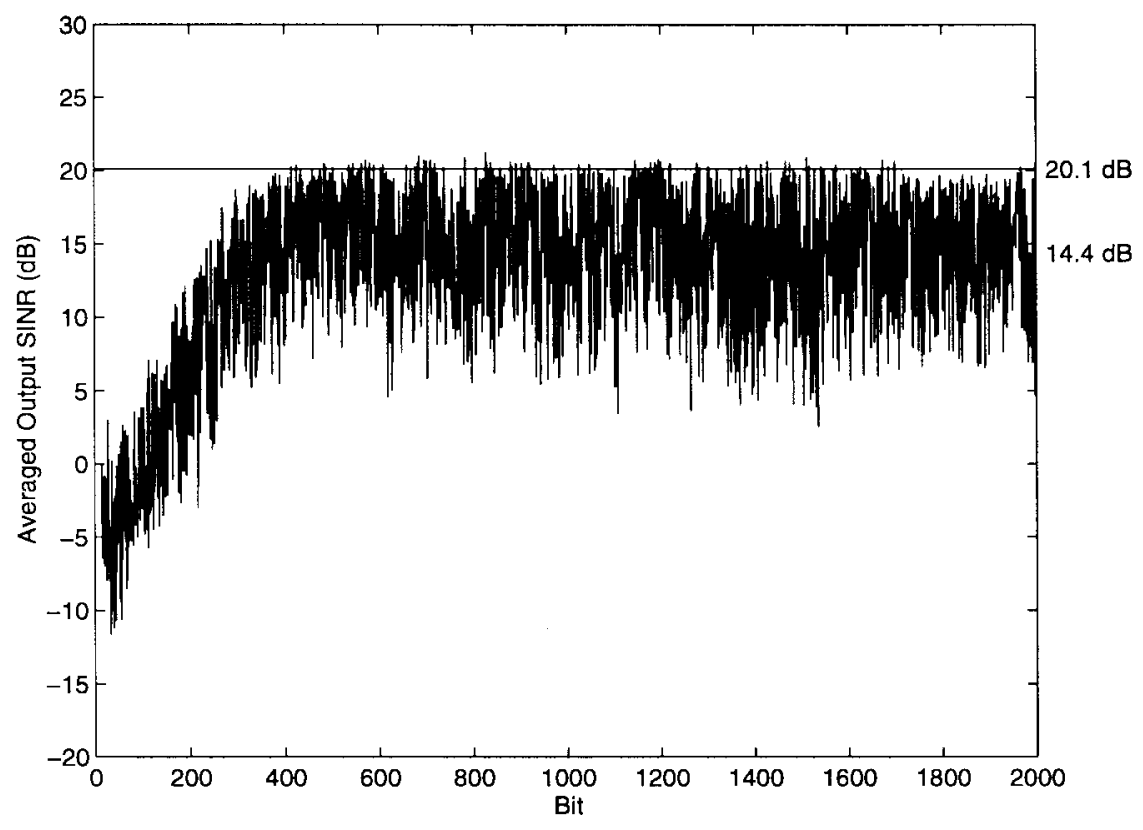

Fig. 5. Performance of the reduced-rank detector in time-invariant AWGN channels, asynchronous users; $N=L=16, m=2, \mathrm{SNR}=22 \mathrm{~dB}$, and $\beta_{1}=0.997$.

with length of $L=6$. The smoothing factor is $m=1$, while the forgetting factors are $\beta_{1}=0.997$ and $\beta_{2}=0.994$. Fig. 2 depicts the desired signal estimation error by the power method (31) and (32), with subspace tracking by PASTd and RO-FST, respectively. The desired signal estimation error is defined as the distance between the subspaces spanned by the true and estimated desired signal vectors given by

$$
d=\left\|\hat{\boldsymbol{u}}_{0}(n) \hat{\boldsymbol{u}}_{0}(n)^{H}-\boldsymbol{u}_{0}(n)\left[\boldsymbol{u}_{0}(n)^{H} \boldsymbol{u}_{0}(n)\right]^{-1} \boldsymbol{u}_{0}(n)^{H}\right\|_{2}
$$

RO-FST significantly outperforms PASTd by this measure. The convergence of PASTd is very slow without special initialization, as observed in [4]. Also, orthonormality of the subspace estimate of PASTd is not guaranteed, unlike RO-FST.

The output SINR performance of four detectors is shown in Figs. 3 and 4 for comparison. The step size in the CMV detector [7] and the decorrelating RAKE (D-RAKE) detector [10] is $\mu=2 \times 10^{-4}$. In the D-RAKE receiver, we use PASTd to estimate the combining vector (the maximal eigenvector of the autocorrelation matrix of the CMV outputs). The CMV detector without channel estimation can lead to low 


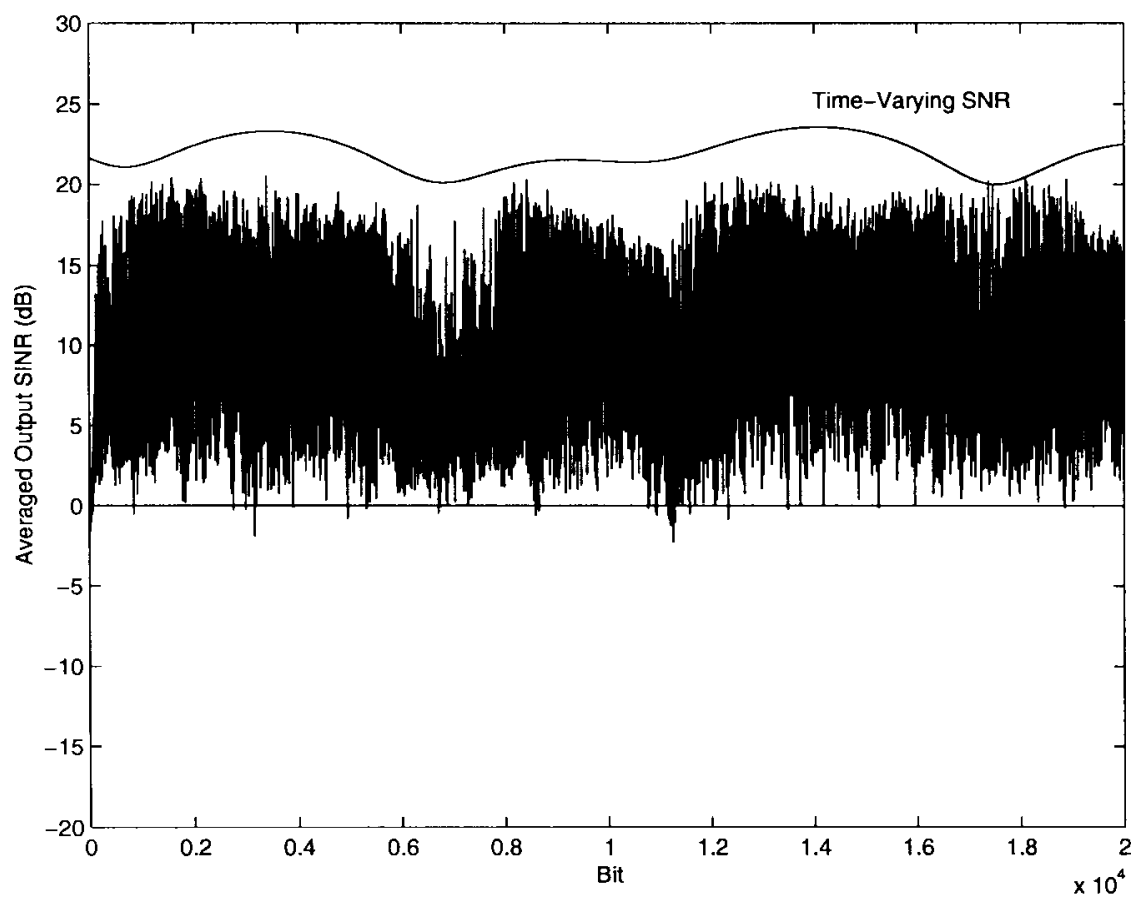

Fig. 6. Performance of the reduced-rank detector in slow-fading channels, $f_{d, \max } T=10^{-4}, L=6, N=16, m=1$, average $\mathrm{SNR}=22 \mathrm{~dB}$, and $\beta_{1}=0.99$.

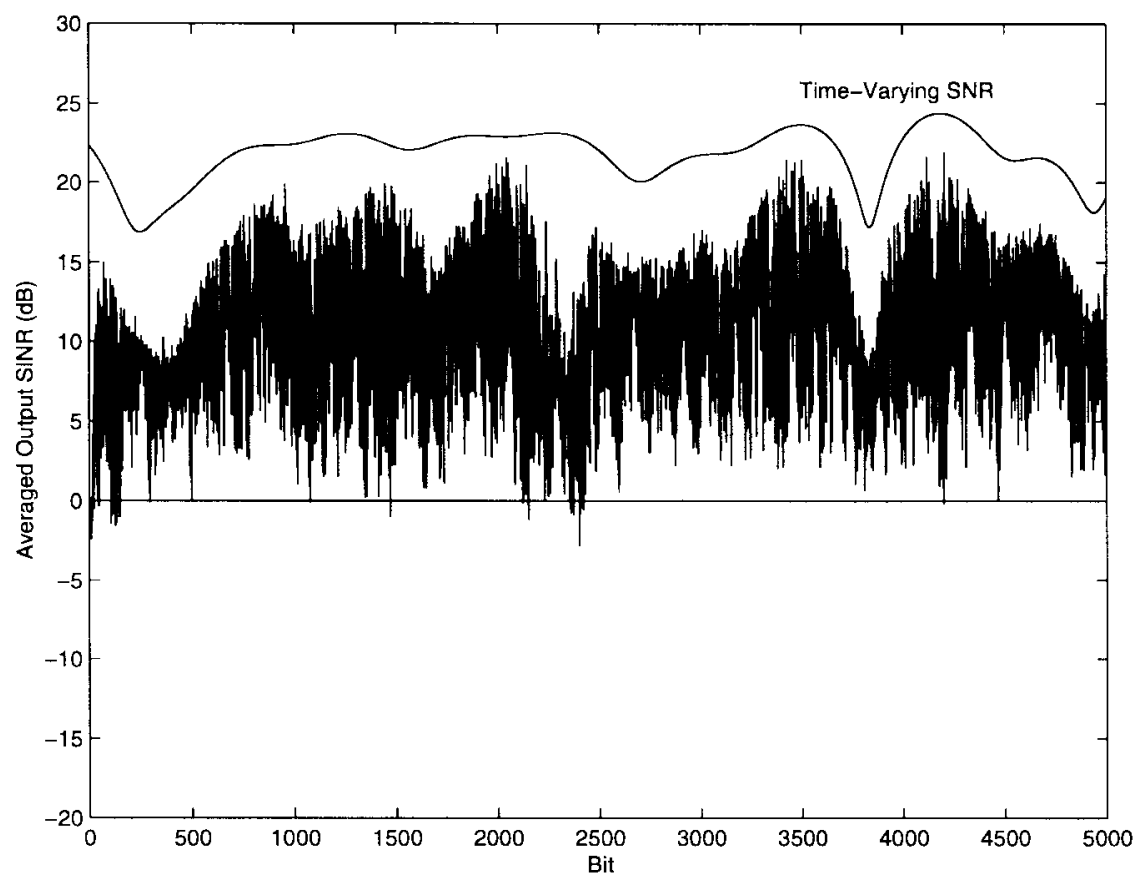

Fig. 7. Performance of the reduced-rank detector in medium-fading channels, $f_{d, \max } T=10^{-3}, L=2, N=16, m=1$, average SNR $=22$ $\mathrm{dB}$, and $\beta_{1}=.099$.

steady state output SINR, since it extracts only part of the desired signal energy [that contributed by $h_{1}(0)$ ]. The DRAKE detector tries to overcome this difficulty by combining outputs of multiple versions of the CMV detector, and it is expected to outperform the latter in the steady state. The combining vector is obtained from the outputs of $\mathrm{CMV}$ detectors, which makes the D-RAKE detector converge slower than the CMV detectors, and the steady-state output SINR limited by the (possibly remarkable) difference between the estimated combining vector and the true channel (optimal combining vector). The subspace MMSE detector by direct inversion of eigenvalues in [4] has the worst performance. Our detector considerably outperforms these detectors due to the improved estimators embodied in the architecture.

Example 2 (Time-Invariant AWGN Channels, Asynchronous Users): In this example, all users (including the desired) have unknown, independent, and uniformly distributed delays and phase shifts. For simplicity, we assume all users are chip synchronized, i.e., the delays are integer multiples of the chip duration. The equivalent channel length is then $L=$ 


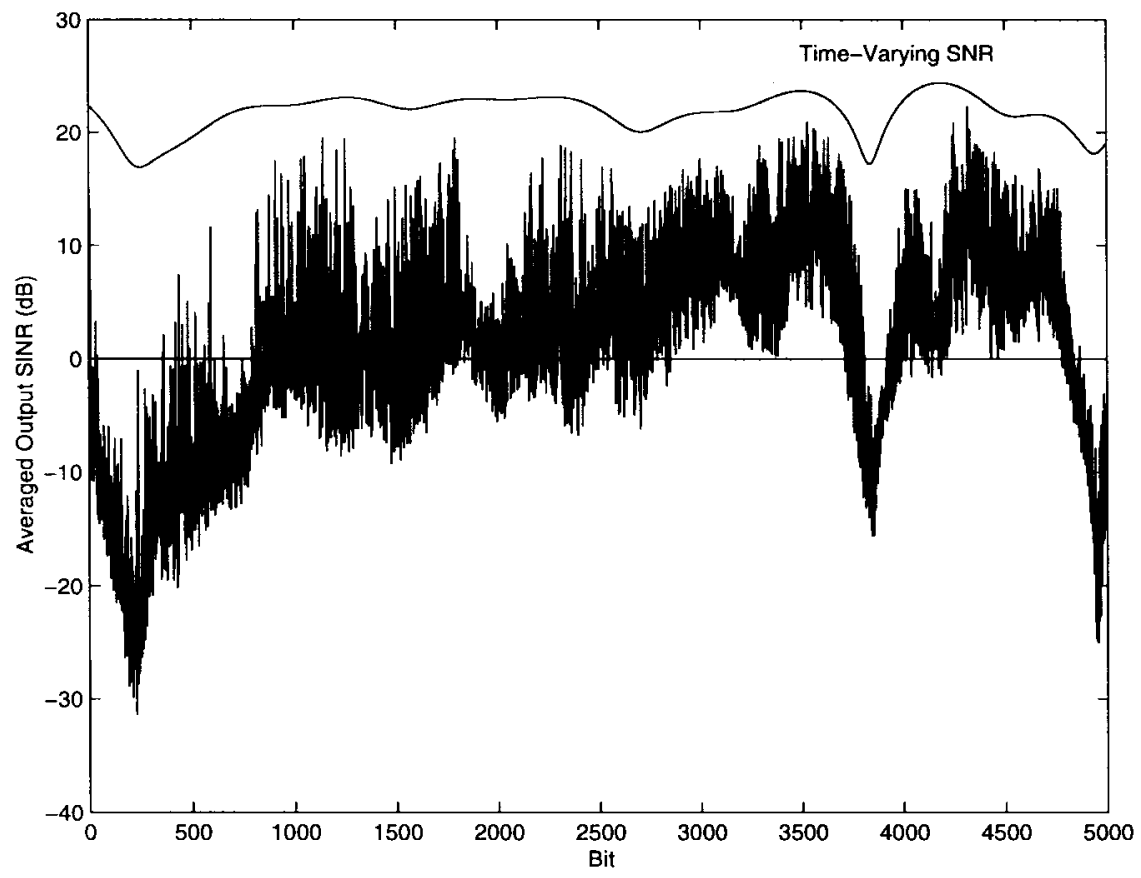

Fig. 8. Performance of the CMV detector in medium-fading channels, $f_{d, \max } T=10^{-3}, L=2, N=16, m=1$, average $\mathrm{SNR}=22 \mathrm{~dB}$, and $\mu=2 \times 10^{-4}$.

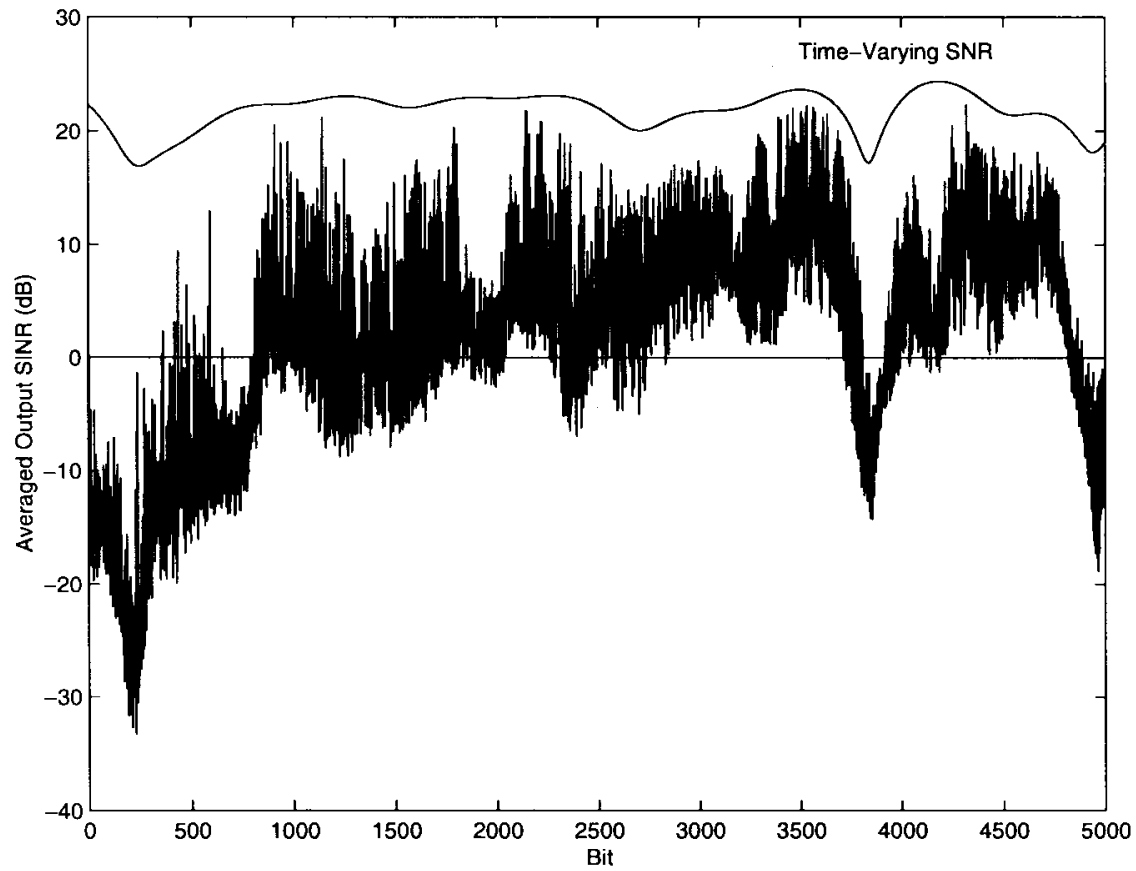

Fig. 9. Performance of the D-RAKE detector in medium-fading channels, $f_{d, \max } T=10^{-3}, L=2, N=16, m=1$, average SNR $=22 \mathrm{~dB}$, and $\mu=2 \times 10^{-4}$

$N=16$, and the smoothing factor is chosen as $m=2$. The output SINR illustrated in Fig. 5 confirms the proposed detector's robustness to user asynchronism, i.e., it is able to implicitly obtain an effective timing estimate for the desired user. To apply the CMV detector [7] in this case, a separate timing estimator must be added to ensure it extracts energy contributed by the nonzero channel coefficient.

Example 3 (Frequency-Selective Slow-Fading Channels): As in Example 1, the data rate is taken as $1 \mathrm{Mbit} / \mathrm{s}$. The maximum Doppler frequency is set to $100 \mathrm{HZ}$ (for a mobile speed of $120 \mathrm{~km} / \mathrm{h}$ and carrier frequency of $900 \mathrm{MHZ}$ ). The channel length is $L=6$ Fig. 6 shows that while the channel variations lead to increased fluctuations in detector output SINR, it almost always remains above $0 \mathrm{~dB}$ under the slowfading conditions assumed in the experiment.

Example 4 (Frequency-Selective Medium-Fading Channels): Performance of the proposed detector in channels with medium-fading rate $\left(f_{d, \max } T=10^{-3}\right)$ is shown in Figs. 7 ( $L$ $=2)$ and $10(L=6)$. For $L=2$ independently fading taps, the detector is able to provide output SINR above $0 \mathrm{~dB}$; however, performance is unacceptable for $L=6$ taps, indicating that the subspace tracking/desired signal estimation degrades very 


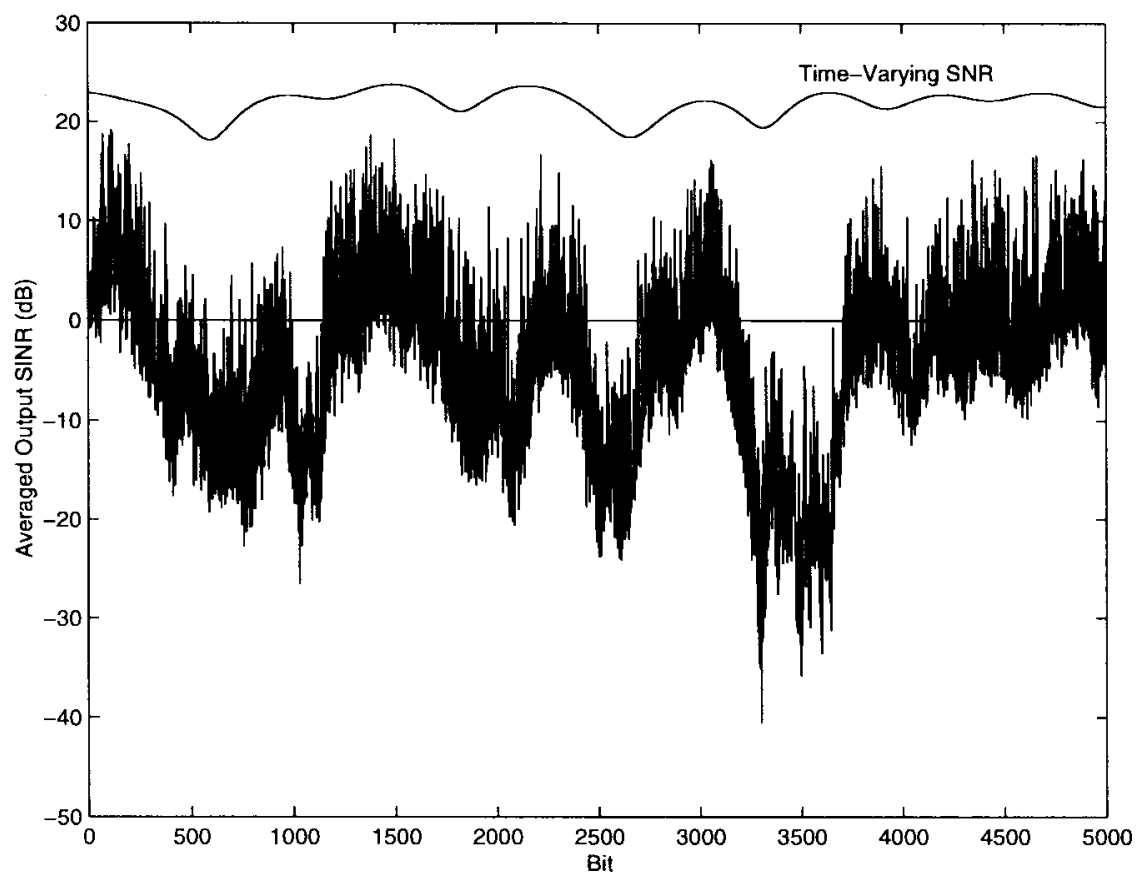

Fig. 10. Performance of the reduced-rank detector in medium-fading channels, $f_{d \text {, } \max } T=10^{-3}, L=6, N=16, m=1$, average SNR $=22$ $\mathrm{dB}$, and $\beta_{1}=0.99$.

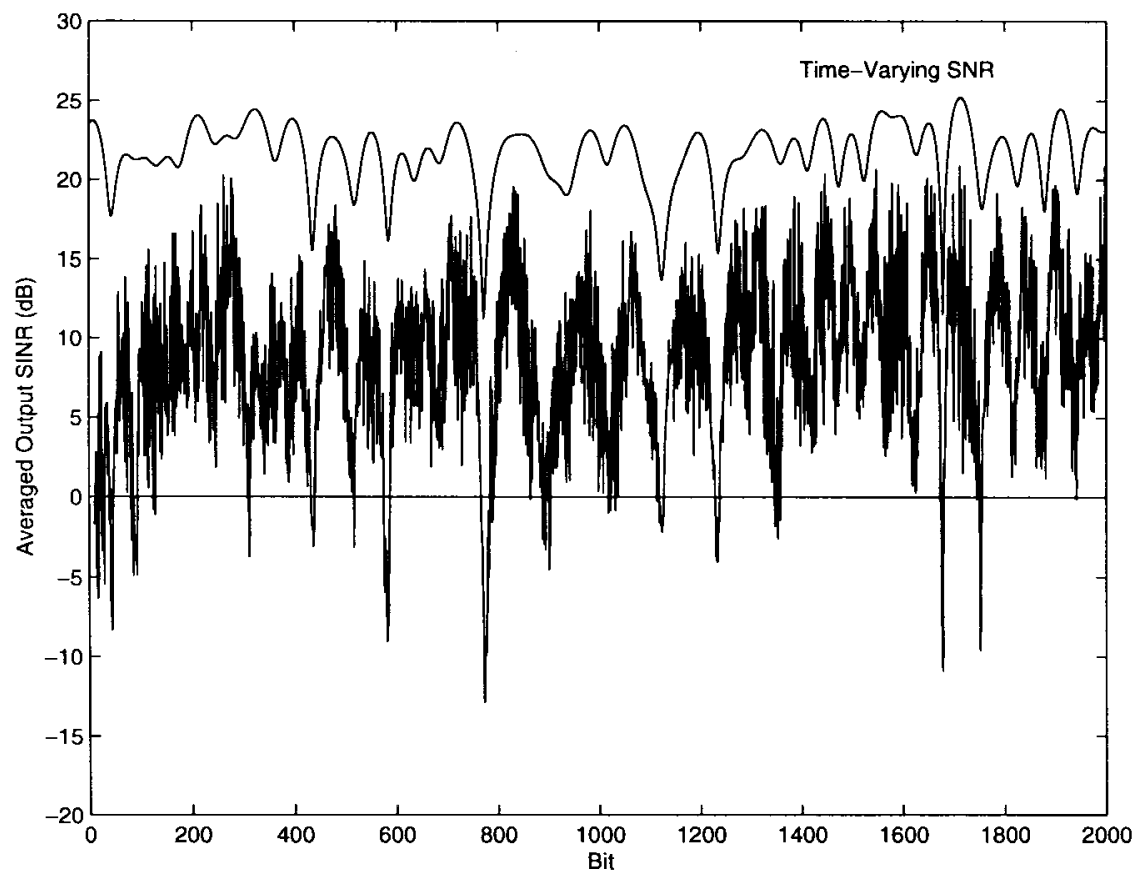

Fig. 11. Performance of the reduced-rank detector in fast-fading channels, $f_{d, \max } T=10^{-2}, L=2, N=16, m=1$, average SNR $=22 \mathrm{~dB}$, and $\beta_{1}=0.99$.

significantly as the number of fading taps increases. The simulation results for $\mathrm{CMV}$ and D-RAKE receivers with $L$ $=2$ in Figs. 8 and 9 show they are clearly inferior to our proposed detector. Note that the D-RAKE detector does not show much improvement over the CMV detector, since it relies on the accuracy of CMV detector output.

Example 5 (Frequency-Selective Fast-Fading Channels): For a maximum fading rate of $f_{d, \max } T=10^{-2}$ considered in our simulation, the output SINR exhibits more excursions below $0 \mathrm{~dB}$ for $L=2$ taps (Fig. 11) as compared to $f_{d \text {, } \max } T$ $=10^{-3}$. The result is similar to an example in [17]. In the time-varying examples, the detector output SINR tend to follow the time-varying SNR due to fading, with significant fluctuations attributable to the blind algorithm (as opposed to decision directed) used in our case.

\section{CONCLUSION}

A new blind adaptive detector for DS-CDMA signals in frequency-selective multipath fading channels is introduced that overcomes some key shortcomings of detectors proposed in the recent literature. The reduced-rank nature of the detector in conjunction with the efficient RO-FST subspace tracker 
leads to only a modest increase in complexity. The detector can accommodate asynchronous channels without any additional timing acquisition mechanism and is able to achieve fast and accurate estimation of the desired user's signature. The detector performance as measured by output SINR reaches acceptable levels typically within a few hundred data symbols. It outperforms some recently proposed blind adaptive multiuser detectors in both convergence speed and steady state SINR especially in slow-fading long channels, and thus is a good candidate for high rate DS-CDMA systems. However, the proposed detector design must be further robustified in fast-fading conditions for adequate performance, probably in combination with effective channel-coding approaches.

\section{ACKNOWLEDGMENT}

The authors would like to thank Dr. L. A. Akers, Director of the Division of Engineering, University of Texas, San Antonio, for his support during the final stages of this work.

\section{REFERENCES}

[1] U. Madhow, "Blind adaptive interference suppression for directsequence CDMA," Proc. IEEE, vol. 86, pp. 2049-2069, Oct. 1998.

[2] M. L. Honig, U. Madhow, and S. Verdú, "Blind adaptive multiuser detection," IEEE Trans. Inform. Theory, vol. 41, pp. 944-960, July 1995.

[3] H. V. Poor and X. Wang, "Code-aided interference suppression for DS/CDMA communications-Part II: Parallel blind adaptive implementations," IEEE Trans. Commun., vol. 45, pp. 1112-1122, Sept. 1997.

[4] X. Wang and H. V. Poor, "Blind multiuser detection: A subspace approach," IEEE Trans. Inform. Theory, vol. 44, pp. 677-690, Mar. 1998.

[5] H. Liu and G. Xu, "A subspace method for signature waveform estimation in synchronous CDMA systems," IEEE Trans. Commun., vol. 44, pp. 1346-1354, Oct. 1996

[6] X. Wang and H. V. Poor, "Blind equalization and multiuser detection in dispersive CDMA channels," IEEE Trans. Commun., vol. 46, pp. 91-103, Jan. 1998.

[7] M. K. Tsatsanis, "Inverse filtering criteria for CDMA systems," IEEE Trans. Signal Processing, vol. 45, pp. 102-112, Jan. 1997.

[8] H. C. Huang and S. Verdú, "Linear differentially coherent multiuser detection for multipath channels," Wireless Personal Commun., vol. 6 , pp. 113-136, 1998.

[9] M. K. Tsatsanis and Z. Xu, "Performance analysis of minimum variance CDMA receivers," IEEE Trans. Signal Processing, vol. 46, pp. 3014-3022, Nov. 1998.

[10] H. Liu and $\mathrm{K}$. Li, "A decorrelating RAKE receiver for CDMA communications over frequency-selective fading channels," IEEE Trans. Commun., vol. 47, pp. 1036-1045, July 1999.

[11] B. Yang, "Projection approximation subspace tracking," IEEE Trans. Signal Processing, vol. 43, pp. 95-107, Jan. 1995.

[12] D. Gesbert, J. Sorelius, and A. Paulraj, "Blind multi-user MMSE detection of CDMA signals," in Proc. ICASSP'98, pp. 3161-3164.

[13] Y. Song and S. Roy, "A blind reduced-rank CDMA detector for multipath channels," in Proc. Eighth CTMC, ICC'99, pp. 62-66.

[14] D. J. Rabideau, "Fast, rank adaptive subspace tracking and applications," IEEE Trans. Signal Processing, vol. 44, pp. 2229-2244, Sept. 1996.
[15] G. H. Golub and C. F. Van Loan, Matrix Computations, 3rd ed. Baltimore, MD: The Johns Hopkins Press, 1996.

[16] Y. Song and S. Roy, "Subspace blind detection of asynchronous CDMA signals in multipath channels," in Proc. SPAWC'99, pp. 21-24.

[17] L. J. Zhu and U. Madhow, "Adaptive interference suppression for direct sequence CDMA over severely time-varying channels," in Proc. Globecom'97.

[18] T. Ojanperä and R. Prasad, "An overview of air interface multiple access for IMT-2000/UMTS,” IEEE Commun. Mag., vol. 36, pp. 82-95, Sept. 1998.

[19] B. N. Parlett, "Remarks on matrix eigenvalue computations," in VLSI and Modern Signal Processing, S. Y. Kung, H. J. Whitehouse, and T. Kailath, Eds. Englewood Cliffs, NJ: Prentice-Hall, 1985, pp. 106-120.

[20] E. Moulines, P. Duhamel, J.-F. Cardoso, and S. Mayrargue, "Subspace methods for the blind identification of multichannel FIR filters," IEEE Trans. Signal Processing, vol. 43, pp. 516-525, Feb. 1995.

[21] G. Mathew, V. U. Reddy, and S. Dasgupta, "Adaptive estimation of eigensubspace," IEEE Trans. Signal Processing, vol. 43, pp. 401-411, Feb. 1995.

[22] S. Roy, "Subspace blind adaptive detection for multi-user CDMA," IEEE Trans. Commun., to be published.

[23] P. Hoeher, "A statistical discrete-time model for the WSSUS multipath channel," IEEE Trans. Vehic. Technol., vol. 41, pp. 461-468, Nov. 1992.

[24] U. Madhow and M. L. Honig, "MMSE interference suppression for direct-sequence spread-spectrum CDMA," IEEE Trans. Commun., vol. 42, pp. 3178-3188, Dec. 1994.

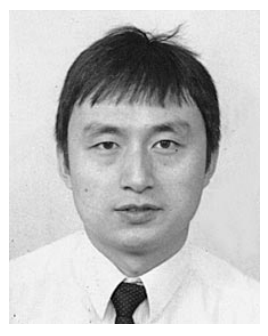

Yu Song (M'98) was born in Chengdu, China, in 1970. He received the B.S. degree from Zhejiang University, Hangzhou, China, in 1989, the M.S degree from the University of Electronics Science and Technology, Chengdu, China, in 1992, and the Ph.D. degree from Tsinghua University, Beijing, China, in 1997, all in electrical engineering.

Since 1997, he has been a Postdoctoral Fellow at the University of Texas, San Antonio. His interests are in digital signal processing, communications, and software.

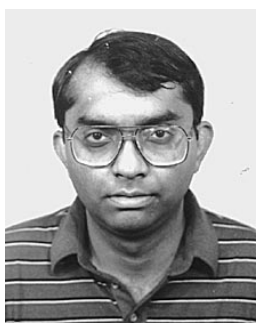

Sumit Roy (S'84-M'88) received the B.Tech. degree from the Indian Institute of Technology, Kanpur, India, in 1983, the M.S. degree in electrical engineering in 1985, the M.A. degree in statistics and applied probability, and the Ph.D. degree in electrical engineering in 1988, all from the University of California, Santa Barbara.

His previous academic appointments were at the Moore School of Electrical Engineering, the University of Pennsylvania, and the University of Texas, San Antonio. He is presently an Associate Professor of Electrical Engineering at the University of Washington, Seattle, where his research interests include the analysis and design of communication systems/networks, with a topical emphasis on next generation mobile/wireless networks.

Dr. Roy's activities for the IEEE Communications Society include membership of several technical committees, and he presently serves as an Editor for the IEEE TRansactions in Communications and the Wireless Series of the IEEE Journal on Selected Areas in Communications. 